\title{
General synthetic approach to 2-phenolic adenine derivatives
}

\author{
Carla Correia, M. Alice Carvalho,* Ashly Rocha and M. Fernanda Proença. \\ Departamento de Química, Universidade do Minho, Campus de Gualtar, 4710-057 Braga, Portugal
}

Fax: +351253678983

E-mail:mac@quimica.uminho.pt.

Received: The date will be inserted once the manuscript is accepted.

Dedication - If you wish to insert a short dedication please overwrite this text, otherwise delete the paragraph.

\begin{abstract}
A simple and general "one pot" procedure for the synthesis of 2,9-diarylpurines with one or multiple hydroxyl groups in the 2- aryl unit is described, from the reaction of 5amino-4-amidinoimidazoles with phenolic aldehydes.
\end{abstract}

Key words: nucleobases, phenols, condensations, aldehydes, ring closure.

Purines have attracted attention of the scientific community mainly due to their biological activity. ${ }^{1}$ During the last decade, purine derivatives were identified as a promising new class of antitubercular agents. The research was focused on the synthesis of nucleoside analogues as siderophore biosynthesis inhibitors, ${ }^{2}$ and on non nucleosides. ${ }^{3}$ In the non nucleoside series, purines having an aryl, a small alkyl or a proton on 9-N were essentially inactive, whereas 9-benzyl-6-(2-furyl)purines, ${ }^{3 \mathrm{~b}, \mathrm{~h}, \mathrm{j}}$ 9-sulfonyl-6mercaptopurines or 6-alkylthiopurines ${ }^{3 e, \mathrm{k}}$ were highly potent. In addition, we recently described the first example of 2,9-diarylpurines (Figure 1) active against Mycobacterium tuberculosis. ${ }^{4}$ The results from biological evaluation showed that the potency of the compounds depends on the substituents in 9-N, 2-C and 6-C of the purine core. The presence of a 4-tolyl group in 9-N and a 3-hydroxyphenyl or a 4hydroxyphenyl substituent in 2-C combined with a secondary amine in 6-C proved to be important features for activity (Figure 1) but a clear structureactivity correlation pattern could not be identified. ${ }^{4}$

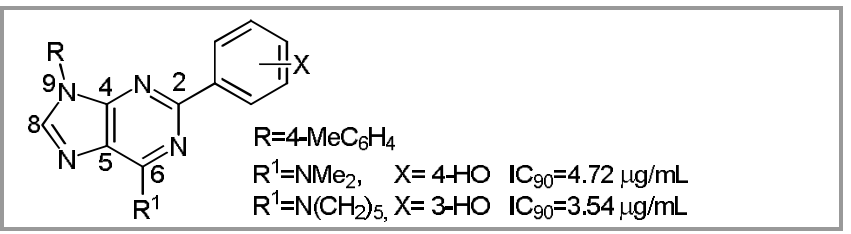

Figure 1 Hit compounds active on Mycobacterium tuberculosis

A number of synthetic methods were developed to incorporate functional groups in the purine core, but costly reagents are usually required. ${ }^{5 a, b}$ In our research group purine derivatives have been obtained by a simple and inexpensive synthetic approach that uses the versatile reactivity of a substituted imidazole. ${ }^{4,5 \mathrm{c}-1}$

The 6-amino-2,9-diarylpurines (Figure 1) are a new promising scaffold active against Mycobacterium tuberculosis and the presence of hydroxyl groups in the aryl subunit in 2-C was considered an important feature for activity. The microorganism needs iron for the biochemical processes and multiple hydroxyl groups in the phenolic subunit could enhance the complexation with the metal. This perception prompted us to develop a new synthetic approach that would allow the efficient synthesis of purines 6 (table 1) having a phenolic subunit in 2-C with two and three hydroxyl substituents. The previous method only allowed the introduction of phenolic units with one hydroxyl group as the reaction with polyphenolic aldehydes, performed in basic medium, was very slow (8-54 days) and extensive degradation occured. ${ }^{4}$

Herein we describe a new and general synthetic approach for 6-amino-9-aryl-2-hydroxy or 2(polyhydroxyphenyl)purines $\mathbf{6}$ using imidazole 1, phenolic aldehydes $\mathbf{2}$ and a cascade of acid and basic catalysis.

When compound 1a (Scheme 1) was combined with 3,4,5-trihydroxybenzaldehyde $\mathbf{2 a}$, in ethanol at $0{ }^{\circ} \mathrm{C}$, using trifluoroacetic acid as catalyst, the starting materials were dissolved and a new white solid precipitated after 5 minutes. This compound was identified as the trifluoroacetate salt of starting material 3. When the reaction was repeated using 2.0 equiv of the acid, a yellow solid precipitated after 4 hours and was identified as $\mathbf{4 a}^{6}$ (Scheme 1).

When imidazole $\mathbf{1 b}$ was reacted with monohydroxy or dihydroxyphenylaldehydes $\mathbf{2 b}$ or $\mathbf{2 c}$, at $6{ }^{\circ} \mathrm{C}$, no solid precipitated from the initial yellow solutions. When the TLC showed the absence of imines 4 , off white solids were isolated and identified as dihydropurines $\mathbf{5 a}, \mathbf{b}^{7 \mathrm{a}}$ (Scheme 1). Dihydropurine $\mathbf{5 c}$ was also isolated in the reaction of imidazole $\mathbf{1 a}$ with 3,4,5trihydroxyphenylaldehyde $\mathbf{2 a}$ when the reaction was carried out at room temperature (Scheme 1). The imine $4 \mathbf{a}$ also evolved to the dihydropurine $\mathbf{5} \mathbf{c}^{\mathrm{7b}}$ after 10 days in ethanolic solution at $8{ }^{\circ} \mathrm{C}($ Scheme 1$)$.

The pure imine 4a showed a single spot on TLC however, by ${ }^{1} \mathrm{H}$ NMR, the spectrum obtained in DMSO solution showed signals consistent with the presence of two compounds in a 7:3 molar ratio. The major compound was identified as $\mathbf{4 a}(70 \%)$ and the minor compound as the dihydropurine 5c (30\%). These results suggested that, in DMSO solution, the imine 4a was cyclizing to the dihydropurine 5c. In order to confirm this result a new ${ }^{1} \mathrm{H}$ NMR spectrum was registered after 2 hours. The spectrum showed once again a mixture of $\mathbf{4 a}$ and $\mathbf{5 c}$ but in a 1:9 molar 


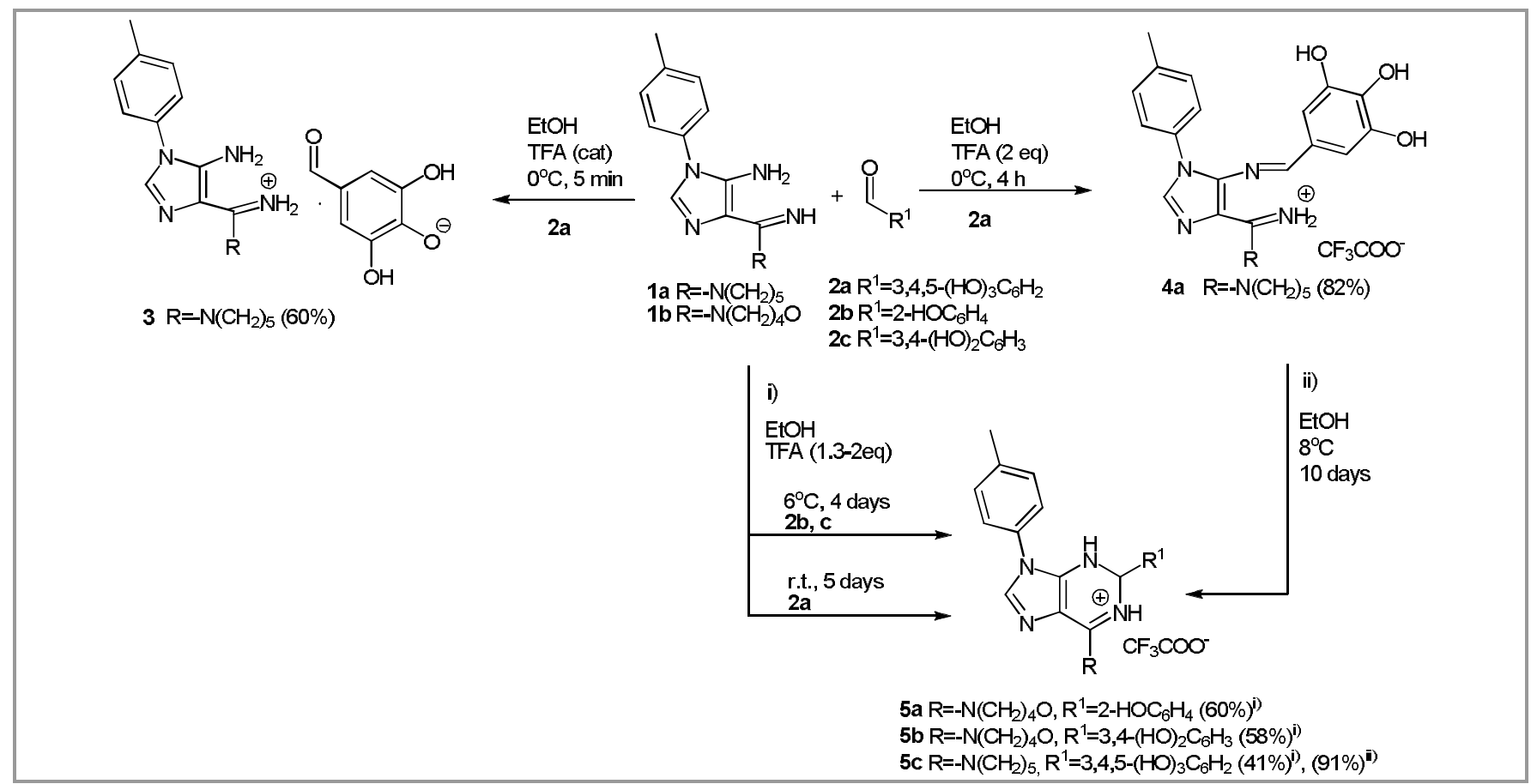

Scheme $\mathbf{1}$ Reactions of imidazoles $\mathbf{1}$ with fenolic aldehydes $\mathbf{2}$ in acidic medium

ratio and this ratio did not change after one week, at room temperature. When the solution was heated in the NMR tube at $60{ }^{\circ} \mathrm{C}$ during 10 minutes a mixture of 4a and 5c was still present, however new signals were observed in the spectrum, assigned to purine $\mathbf{6 i}$, and to the imidazole $\mathbf{1 a}$ and aldehyde $\mathbf{2 a}$, the starting reagents. The hydrolysis of the imine $\mathbf{4 a}$ was also observed by ${ }^{1} \mathrm{H}$ NMR in an acidified solution of $\mathbf{4 a}$ in DMSO-d6. These results indicate that in DMSO solution, at room temperature, the imine $\mathbf{4 a}$ evolves rapidly to the dihydropurine $\mathbf{5 c}$ leading to an equilibrium mixture. Under heating, compound 5c evolves to generate the purine 6i. However, in acid

Table 1 Synthesis of purines 6

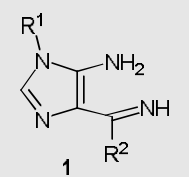

Imidazole (1) $\mathrm{R}^{1} \quad \mathrm{R}^{2}$

1 b $4-\mathrm{MeC}_{6} \mathrm{H}_{4}$

1b $4-\mathrm{MeC}_{6} \mathrm{H}_{4}$

1b $\quad 4-\mathrm{MeC}_{6} \mathrm{H}_{4}$

1 b $4-\mathrm{MeC}_{6} \mathrm{H}_{4}$

1 b $4-\mathrm{MeC}_{6} \mathrm{H}_{4}$

1 b $4-\mathrm{MeC}_{6} \mathrm{H}_{4}$

1 b $4-\mathrm{MeC}_{6} \mathrm{H}_{4}$

$1 a \quad 4-\mathrm{MeC}_{6} \mathrm{H}_{4}$

$1 a \quad 4-\mathrm{MeC}_{6} \mathrm{H}_{4}$

1c $4-\mathrm{MeC}_{6} \mathrm{H}_{4}$

1c $4-\mathrm{MeC}_{6} \mathrm{H}$

$$
\mathrm{R}^{2} \quad \text { Aldehyde (2) }
$$

$-\mathrm{N}\left(\mathrm{CH}_{2}\right)_{4} \mathrm{O} \quad$ 2b $\quad 2-\mathrm{HOC}_{6} \mathrm{H}_{4}$

$-\mathrm{N}\left(\mathrm{CH}_{2}\right)_{4} \mathrm{O} \quad$ 2d $\quad 3-\mathrm{HOC}_{6} \mathrm{H}_{4}$

$-\mathrm{N}\left(\mathrm{CH}_{2}\right)_{4} \mathrm{O} \quad$ 2e $\quad 4-\mathrm{HOC}_{6} \mathrm{H}_{4}$

$-\mathrm{N}\left(\mathrm{CH}_{2}\right)_{4} \mathrm{O} \quad$ 2f $\quad 2,3-(\mathrm{HO})_{2} \mathrm{C}_{6} \mathrm{H}_{3}$

$-\mathrm{N}\left(\mathrm{CH}_{2}\right)_{4} \mathrm{O} \quad 2 \mathrm{c} \quad 3,4-(\mathrm{HO})_{2} \mathrm{C}_{6} \mathrm{H}_{3}$

$-\mathrm{N}\left(\mathrm{CH}_{2}\right)_{4} \mathrm{O} \quad 2 \mathrm{~g} \quad 2,3,4-(\mathrm{HO})_{3} \mathrm{C}_{6} \mathrm{H}_{2}$

$-\mathrm{N}\left(\mathrm{CH}_{2}\right)_{4} \mathrm{O} \quad \mathbf{2 a} \quad 3,4,5-(\mathrm{HO})_{3} \mathrm{C}_{6} \mathrm{H}_{2}$

$-\mathrm{N}\left(\mathrm{CH}_{2}\right)_{5} \quad 2 \mathrm{c} \quad 3,4-(\mathrm{HO})_{2} \mathrm{C}_{6} \mathrm{H}_{3}$

$-\mathrm{N}\left(\mathrm{CH}_{2}\right)_{5}$

2 a $\quad 3,4,5-(\mathrm{HO})_{3} \mathrm{C}_{6} \mathrm{H}_{2}$

$-\mathrm{N}\left(\mathrm{CH}_{2}\right)_{4} \mathrm{~S} \quad 2 \mathrm{a} \quad 3,4,5-(\mathrm{HO})_{3} \mathrm{C}_{6} \mathrm{H}_{2}$

$-\mathrm{N}\left(\mathrm{CH}_{2}\right)_{4} \mathrm{~S} \quad 2 \mathrm{~g} \quad 2,3,4-(\mathrm{HO})_{3} \mathrm{C}_{6} \mathrm{H}_{2}$

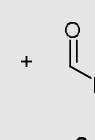

2

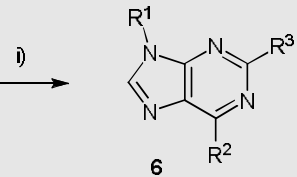

Reaction conditions

product Yield

i) a) $\mathbf{1 b}(0.12 \mathrm{~g}), \mathbf{2 b}(1.1 \mathrm{eq}$ ), EtOH, TFA ( $1.3 \mathrm{eq}), 11^{\circ} \mathrm{C}, 1$ day $\mathbf{6 a}$ b) $\operatorname{DMSO}(0.2 \mathrm{~mL}), \mathrm{Et}_{3} \mathrm{~N}(10 \mathrm{eq}), 40^{\circ} \mathrm{C}, 2$ days

i) a) $\mathbf{1 b}(0.12 \mathrm{~g}), \mathbf{2 d}(1.1 \mathrm{eq}$.$) , EtOH, TFA (1.3 \mathrm{eq}), 11^{\circ} \mathrm{C}, 7$ days $\quad \mathbf{6 b} \quad 79$

b) $\operatorname{DMSO}(0.2 \mathrm{~mL}), \mathrm{Et}_{3} \mathrm{~N}(10 \mathrm{eq}), 40^{\circ} \mathrm{C}, 2$ days

i) a) $\mathbf{1 b}(0.13 \mathrm{~g})$, 2e (1.1 eq. $)$, EtOH, TFA $(1.3 \mathrm{eq}), 11^{\circ} \mathrm{C}, 1$ day $\mathbf{6 c} \quad 78$

b) $\operatorname{DMSO}(0.2 \mathrm{~mL}), \mathrm{Et}_{3} \mathrm{~N}(10 \mathrm{eq}), 40^{\circ} \mathrm{C}, 2$ days

i) a) $\mathbf{1 b}(0.17 \mathrm{~g}), \mathbf{2 f}(1.1$ eq.), EtOH), TFA $(2.0 \mathrm{eq})$, r.t., $30 \mathrm{~min} \quad \mathbf{6 d} \quad 88$

b) $\mathrm{DMSO}, \mathrm{EtOH}, \mathrm{Et}_{3} \mathrm{~N}(10$ eq $), 40^{\circ} \mathrm{C}, 6$ days

i) a) $\mathbf{1 b}(0.12 \mathrm{~g}), \mathbf{2 c}(1.1 \mathrm{eq}$.$) , EtOH, TFA (1.3 \mathrm{eq}), 11^{\circ} \mathrm{C}, 1$ day $\quad \mathbf{6 e} \quad 58$

b) $\mathrm{DMSO}, \mathrm{Et}_{3} \mathrm{~N}(10 \mathrm{eq}), 40^{\circ} \mathrm{C}, 4$ days

i) a) $\mathbf{1 b}(0.30 \mathrm{~g}), \mathbf{2 g}(1.1 \mathrm{eq}),. \operatorname{EtOH}, \operatorname{TFA}(1.3 \mathrm{eq})$, r.t., 1 day $\quad$ 6f $\quad 59$

b) $\mathrm{DMSO}, \mathrm{Et}_{3} \mathrm{~N}(10 \mathrm{eq}), 40^{\circ} \mathrm{C}, 6$ days

i) a) $\mathbf{1 b}(0.28 \mathrm{~g}), \mathbf{2 a}(1.1$ eq.), EtOH, TFA (1.3 eq), r.t., 6 days $\quad \mathbf{6 g} \quad 50$

b) DMSO, $\mathrm{Et}_{3} \mathrm{~N}(10 \mathrm{eq}), 40^{\circ} \mathrm{C}, 1$ day

i) a) $\mathbf{1 a}(0.21 \mathrm{~g}), \mathbf{2 c}(1.1 \mathrm{eq}$ ), EtOH, TFA (1.5 eq), r.t., 6 days $\quad \mathbf{6 h} \quad 57$

b) $\mathrm{DMSO}, \mathrm{Et}_{3} \mathrm{~N}(10 \mathrm{eq}), 40^{\circ} \mathrm{C}, 4$ days

i) a) $\mathbf{1 a}(0.21 \mathrm{~g}), \mathbf{2 a}(1.1 \mathrm{eq}$.$) , EtOH, TFA (1.5 eq), r.t., 10$ days $\quad \mathbf{6 i} \quad 57$

b) $\mathrm{DMSO}, \mathrm{Et}_{3} \mathrm{~N}(10 \mathrm{eq}), 80^{\circ} \mathrm{C}, 1$ day

i) a) $\mathbf{1 c}(0.16 \mathrm{~g}), \mathbf{2 a}(1.1$ eq.), EtOH, TFA (1.3 eq), r.t., 5 days $\quad \mathbf{6 j} \quad 67$

b) $\mathrm{DMSO}_{2} \mathrm{Et}_{3} \mathrm{~N}(10 \mathrm{eq}), 80^{\circ} \mathrm{C}, 1$ day

i) a) $\mathbf{1 c}(0.11 \mathrm{~g}), \mathbf{2 g}(1.1 \mathrm{eq}$.$) , EtOH, TFA (1.3 eq), r.t., 4$ days $\quad \mathbf{6 k}$

60

b) $\mathrm{DMSO}, \mathrm{Et}_{3} \mathrm{~N}(10 \mathrm{eq}), 40^{\circ} \mathrm{C}, 5$ days 
solution, hydrolysis of the imine regenerates the starting materials 1a and $\mathbf{2 a}$.

The dihydropurine 5c was also solubilised in DMSO$d 6$ and the ${ }^{1} \mathrm{H}$ NMR spectrum, acquired after 5 minutes, showed that $\mathbf{5 c}$ was the only product present in solution. However, after the addition of triethylamine and heating at $80{ }^{\circ} \mathrm{C}$ for 20 hours only purine $6 \mathbf{i}$ was present in solution.

Considering that the reaction of imidazoles $\mathbf{1}$ with aldehydes 2, in ethanol in the presence of 1.3-2 equiv of TFA led to imines $\mathbf{4}$ and dihydropurines $\mathbf{5}$, and that compounds $\mathbf{4}$ and $\mathbf{5}$ in DMSO solution, under heating, and in the presence of triethylamine generated the desired purine 6, a new "one pot" synthetic procedure to generate purines $\mathbf{6}^{8}$ was designed, starting from imidazoles $\mathbf{1}$ and aldehydes $\mathbf{2}$. The reaction was initially carried out in ethanol and acid (1.3-2 equiv) until total consumption of the starting materials (by TLC). The solvent was then removed in the rotary evaporator and the reactions proceeded in DMSO and base, with heating.

These experimental conditions were applied to the reaction of imidazoles 1a-c with aldehydes $\mathbf{2 a - g}$, having one, two or three hydroxyl groups. The purines 6a-k were obtained directly from the reaction mixture after 2-10 days (Table 1). When degradation was observed, the pure products were isolated in lower yields after dry flash chromatography (compounds $6 \mathbf{6}-$ h).

In summary this work describes a new versatile and simple "one-pot" method to synthesize 6-amino-9substituted-2-hydroxy or 2(polyhydroxyphenyl)purines 6 starting from imidazoles $\mathbf{1}$ and phenolic aldehydes $\mathbf{2}$. The formation of the imine intermediate, is favoured in ethanol using acid catalysis, however the cyclization and oxidation, to generate the purine core, is favoured in dimethylsulfoxide, under heating, using base catalysis.

\section{Acknowledgements}

The NMR spectrometer (Bruker 400 Avance III) is part of the National NMR Network, supported with funds from Fundação para a Ciência e a Tecnologia (FCT). The authors gratefully acknowledge the financial support by F.C.T. (project $n^{\circ} \mathrm{F}-$ COMP-01-0124-FEDER-022716 (ref. FCT PEst/QUI/UI0686/2011) FEDER-COMPETE, FCT-Portugal and the PhD grant to C. Correia (SFRH/BD/22270/2005).

\section{References and notes}

(1) Legraverend, M.; Grierson, D. S. Bioorg. Med. Chem. 2006, 14, 3987.

(2) a) Gupte, A.; Boshoff, H. I.; Wilson, D. J.; Neres, J.; Labello, N. P.; Somu, R. V.; Xing, C.; Barry III, C. E.; Aldrich, C. C. J. Med. Chem., 2008, 51 (23), 7495; b) Neres, J.; Labello, N. P.; Somu, R. V.; Boshoff, H. I.; Wilson, D. J.; Vannada, J.; Chen L.; Barry III, C. E.; Bennett, E. M.; Aldrich, C. C. J. Med. Chem., 2008, 51
(17), 5349; c) Long, M. C.; Shaddix, S. C.; MoukhaChafiq, O.; Maddry, J. A.; Nagy, L.; Parker, W. B. Biochem. Pharmacol. 2008, 75, 1588; d) Bisseret, P.; Thielges, S.; Bourg, S.; Miethke, M.; Marahiel, M. A.; Eustachea, J. Tetrahedron Letters 2007, 48, 6080; e) Qiao, C.; Gupte, A.; Boshoff, H. I.; Wilson, D. J.; Bennett, E. M.; Somu, R.V.; Barry, C. E.; Aldrich, C. C. J. Med. Chem., 2007, 50 (24), 6080; f) Rai, D.; Johar, M.; Srivastav, N. C.; Manning, T.; Agrawal, B.; Kunimoto, D. Y.; Kumar, R. J. Med. Chem., 2007, 50 (19), 4766; g) Qiao, C.; Wilson, D. J.; Bennett, E. M.; Aldrich, C. C. J. Am. Chem. Soc., 2007, 129 (20), 6350; h) Vannada, J.; Bennett, E. M.; Wilson, D. J.; Boshoff, H. I.; Barry, C. E.; Aldrich, C. C. Org. Lett., 2006, 8 (21), 4707; i) Somu, R.V.; Wilson, D. J.; Bennett, E. M.; Boshoff, H. I.; Celia, L.; Beck, B. J.; Barry, C. E.; Aldrich, C. C. J. Med. Chem., 2006, 49 (26), 7623; j) Bennett, E. M.; Barry, C.E.; Aldrich, C. C. J. Med. Chem., 2006, 49 (1), 31.

(3) a) Bakkestuen, A. K.; Gundersen, L.-L; Utenova, B. T. J. Med. Chem. 2005, 48, 2710; b) Braendvang, M.; Gundersen, L.-L. Bioorg. Med. Chem. 2007, 15, 7144;

c) Braendvang, M.; Gundersen, L.-L. Bioorg. Med. Chem. 2005, 13, 6360; d) Bakkestuen, A. K.;

Gundersen, L.-L.; Petersen, D.; Utenova, B. T.; Vik, A. Org. Biomol. Chem. 2005, 3, 1025; e) Pathak, A. K.; Pathak, V.; Seitz, L.E.; Suling, W. J.; Reynolds, R.C. J. Med. Chem., 2004, 47 (1), 273; f) Barrow, E. W.; Westbrook, L.; Bansal, N.; Suling, W. J.; Maddry, J. A.; Parker, W. B.; Barrow, W. W. J. Antimicrob. Chemother., 2003, 52, 801; g) Andersen, G.; Gundersen, L.-L.;

Nissen-Meyer, J.; Rise, F.; Spilsberg, B. Bioorg. Med. Chem. Lett., 2002, 12, 567; h) Gundersen, L.-L.; NissenMeyer, J.; Spilsberg, B. J. Med. Chem. 2002, 45, 1383; i) Scozzafava, A.; Mastrolorenzo, A.; Supuran, C. T. Bioorg. Med. Chem Lett. 2001, 11, 1675; j) Bakkestuen, A. K.; Gundersen, L.-L.; Langli, G.; Liu, F.; Nolsoe, J. M. J. Bioorg. Med. Chem Lett. 2000, 10, 1207; k) Scozzafava, A.; Mastrolorenzo, A.; Supuran, C. T.; Bioorg. Med. Chem. Lett. 2001, 11, 1675.

(4) Correia, C.; Carvalho, M. A.; Proença, M. F. Tetrahedron, 2009, 65, 6903.

(5) a) For a recent review see Legraverend, M. Tetrahedron 2008, 64, 8585; b) Ibrahim, N.; Legraverend, M. J. Org. Chem., 2009, 74 (1), 463; c) Alves, M. J.; Booth, B. L.; Freitas, A. P.; Proença, M. F. J. Chem. Soc. Perkin Trans. 1 1992, 913; d) Booth, B. L.; Dias, A. M.; Proença, M. F. J. Chem. Soc. Perkin Trans. 1 1992, 2119; e) Alves, M. J.; Booth, B. L.; Proença , M. F. J. Heterocycl. Chem. 1994, 31, 345; f) Booth, B. L.; Coster, R. D.; Proença, M. F. Synthesis 1988, 389; g) Alves, M. J.; Booth, B. L.; Carvalho, M. A.; Pritchard, R. G.; Proença, M. F. J. Heterocycl. Chem. 1997, 34, 739; h) Al-Azmi, A.; Booth, B. L.; Carpenter, R. A.; Carvalho, M. A.; Marrelec, E.; Pritchard, R. G.; Proença, M. F. J. Chem. Soc. Perkin Trans. 1 2001, 2532; i) Booth, B. L.; Cabral, I. M.; Dias, A. M.; Freitas, A. P.; Matos-Beja, A. M.; Proença, M. F.; Ramos-Silva, M. J. Chem. Soc. Perkin Trans. 1 2001, 1241; j) Carvalho, M. A.; Esteves, T. M.; Proença, M. F.; Booth, B. L. Org. Biomol. Chem. 2004, 2, 1019; k) Carvalho, M. A.; Álvares, Y.; Zaki, M. E.; Proença, M. F.; Booth, B. L. Org. Biomol. Chem. 2004, 2, 2340; 1) Alves, M. J.; Carvalho, M. A.; Carvalho, S.; Dias, A. M.; Fernandes, F. H.; Proença, M. F. Eur. J. Org. Chem. 2007, 4881.

(6) Procedure for the synthesis of imine 4a (Scheme 1): To a stirred suspension of imidazole $1 \mathbf{a}(0.14 \mathrm{~g}, 0.49$ $\mathrm{mmol})$ in ethanol $(0.4 \mathrm{~mL}), 1.1$ equiv of aldehyde $\mathbf{2 a}$ $(0.09 \mathrm{~g}, 1.1$ equiv) and 2 equiv of TFA $(75 \mu \mathrm{L})$ were 
added, at $0{ }^{\circ} \mathrm{C}$. A yellow solution developed and a yellow solid started to precipitate after 25 minutes. When the TLC indicated the absence of starting material $(4 \mathrm{~h})$, ethanol was added $(0.8 \mathrm{~mL})$ and the yellow solid was filtered. The solid was washed with ethanol and diethyl ether and identified as compound $4 \mathbf{a}(0.22 \mathrm{~g}$, $0.40 \mathrm{mmol}, 82 \%$ ). mp $124-126^{\circ} \mathrm{C}$. IR (Nujol mull): $3498,3352,3210,1665,1626,1604,1586,1521 \mathrm{~cm}^{-1}$. ${ }^{1} \mathrm{H}$ NMR (300 MHz, DMSO-d6): $\delta=1.62\left(\mathrm{~s}, 6 \mathrm{H}, \mathrm{CH}_{2}\right)$, 2.37 (s, $\left.3 \mathrm{H}, \mathrm{CH}_{3}\right), 3.58$ (br s, $\left.4 \mathrm{H}, \mathrm{CH}_{2}\right), 6.82$ (s, $2 \mathrm{H}, \mathrm{Ar}-$ $\mathrm{H}), 7.30-7.45(\mathrm{~m}, 4 \mathrm{H}, \mathrm{Ar}-\mathrm{H}), 8.17(\mathrm{~s}, 2 \mathrm{H}, 2-\mathrm{H}, \mathrm{N}=\mathrm{CH})$, 8.80-9.60 (m, 5H, NH, HO, $\mathrm{D}_{2} \mathrm{O}$ exchangeable). Anal. calcd for $\mathrm{C}_{23} \mathrm{H}_{25} \mathrm{~N}_{5} \mathrm{O}_{3} \cdot \mathrm{CF}_{3} \mathrm{COOH} \cdot \mathrm{H}_{2} \mathrm{O}: \mathrm{C}, 54.44 ; \mathrm{H}$, $5.08 ; \mathrm{N}, 12.70$. Found: C, 54.68; H, 5.41; N, 12.45.

(7) Procedure for the synthesis of dihydropurines 5c(Scheme 1): Method i) Aldehyde 2a (0.07 g, 1.0 equiv) and TFA ( $40 \mu \mathrm{L}, 1.3$ equiv) were added to a stirred suspension of imidazole $1 \mathrm{a}(0.11 \mathrm{~g}, 0.40 \mathrm{mmol})$ in ethanol $(2.0 \mathrm{~mL})$, at room temperature. The yellow solution became light yellow and when TLC showed the absence of imine 4 ( 5 days), the solution was concentrated in the rotary evaporator. The off-white solid was filtered, washed with ethanol and diethyl ether and identified as compound $\mathbf{5 c}(0.09 \mathrm{~g}, 0.16 \mathrm{mmol}, 41$ $\%)$. Method ii) A yellow ethanolic solution of $4 \mathbf{a}(0.09$ $\mathrm{g}, 0.16 \mathrm{mmol}$ ) was stirred at $8{ }^{\circ} \mathrm{C}$ until TLC showed the absence of starting material (10 days). The solution was concentrated in the rotary evaporator leading to an offwhite solid that was filtered and washed with diethyl ether and identified as compound $\mathbf{5 c}(0.08 \mathrm{~g}, 0.15 \mathrm{mmol}$, 91\%). mp 218-220 ${ }^{\circ} \mathrm{C}$. IR (Nujol mull): 3535,3202 , 1679, 1613, $1530 \mathrm{~cm}^{-1} .{ }^{1} \mathrm{H}$ NMR (300 MHz, DMSO$d 6): \delta=1.69\left(\mathrm{~s}, 6 \mathrm{H}, \mathrm{CH}_{2}\right), 2.39\left(\mathrm{~s}, 3 \mathrm{H}, \mathrm{CH}_{3}\right), 3.70(\mathrm{~m}$, $\left.4 \mathrm{H}, \mathrm{CH}_{2}\right), 5.71(\mathrm{t}, 1 \mathrm{H}, J=4.8 \mathrm{~Hz}, 2-\mathrm{H}), 6.28(\mathrm{~s}, 2 \mathrm{H}, \mathrm{Ar}-$ $\mathrm{H}), 7.40-7.46(\mathrm{~m}, 4 \mathrm{H}, \mathrm{Ar}-\mathrm{H}), 7.88(\mathrm{~s}, 1 \mathrm{H}, 8-\mathrm{H}), 8.07(\mathrm{~d}$, $1 \mathrm{H}, J=4.8 \mathrm{~Hz}, \mathrm{NH}, \mathrm{D}_{2} \mathrm{O}$ exchangeable), 8.23 (br s, $1 \mathrm{H}$, $\mathrm{HO}, \mathrm{D}_{2} \mathrm{O}$ exchangeable), $8.88(\mathrm{~d}, 1 \mathrm{H}, J=4.8 \mathrm{~Hz}, \mathrm{NH}$, $\mathrm{D}_{2} \mathrm{O}$ exchangeable), $8.99\left(\mathrm{~s}, 2 \mathrm{H}, \mathrm{HO}, \mathrm{D}_{2} \mathrm{O}\right.$ exchangeable). ${ }^{13} \mathrm{C}$ NMR (75 MHz, DMSO-d6): $\delta=$ $20.62\left(\mathrm{CH}_{3}\right), 23.32,25.80,48.82,64.77$ (2-C), 105.12, $110.60,124.14,129.16,130.32,130.88,133.39,135.14$ (8-C), 138.65, 145.73, 145.84, 150.00. Anal. calcd for $\mathrm{C}_{23} \mathrm{H}_{25} \mathrm{~N}_{5} \mathrm{O}_{3} \cdot \mathrm{CF}_{3} \mathrm{COOH} \cdot 2.1 \mathrm{H}_{2} \mathrm{O}: \mathrm{C}, 52.56 ; \mathrm{H}, 5.29 ; \mathrm{N}$, 12.26. Found: C, 52.55; H, 5.10; N, 12.07 .

(8) Procedure for the synthesis of purine $6 f$ (Table 1): Aldehyde $2 \mathrm{~g}$ ( $0.18 \mathrm{~g}, 1.1$ equiv) and TFA $(166 \mu \mathrm{L}, 1.3$ equiv) were added to a stirred suspension of imidazole $1 \mathbf{b}(0.30 \mathrm{~g}, 1.08 \mathrm{mmol})$ in ethanol $(0.3 \mathrm{~mL})$, at room temperature during 1 day. Then, the solvent was removed in the rotary evaporator, and DMSO $(0.3 \mathrm{~mL})$ was added to the crude solid followed by triethylamine $(1.35 \mathrm{~mL}, 10$ equiv) and the reaction was stirred at 40 ${ }^{\circ} \mathrm{C}$ during 6 days. Addition of water and cooling for 10 minutes led to a brown solid that was filtered and washed with water and diethyl ether $(0.42 \mathrm{~g})$. The brown solid was purified by dry flash chromatography on silica gel using $500 \mathrm{~mL}$ of diethyl ether as eluent to give an off-white solid identified as $6 \mathbf{6}(0.25 \mathrm{~g}, 0.60 \mathrm{mmol}$, $59 \%$ ). $\mathrm{mp}>300{ }^{\circ} \mathrm{C}$. IR (Nujol mull): $3550,3465,3337$, $3101,1637,1624,1581,1528 \mathrm{~cm}^{-1}$. ${ }^{1} \mathrm{H}$ NMR (300 MHz, DMSO-d6): $\delta=2.41\left(\mathrm{~s}, 3 \mathrm{H}, \mathrm{CH}_{3}\right), 3.85(\mathrm{~s}, 4 \mathrm{H}$, $\left.\mathrm{CH}_{2}\right), 4.30\left(\mathrm{br} \mathrm{s}, 4 \mathrm{H}, \mathrm{CH}_{2}\right), 6.36(\mathrm{~d}, 1 \mathrm{H}, J=9.0 \mathrm{~Hz}, \mathrm{Ar}-$ H), 7.43 (d, 2H, $J=8.4, \mathrm{Ar}-\mathrm{H}), 7.64(\mathrm{~d}, 2 \mathrm{H}, J=8.7 \mathrm{~Hz}$, Ar-H), 7.68 (d, $1 \mathrm{H}, J=9.0 \mathrm{~Hz}, \mathrm{Ar}-\mathrm{H}), 8.24$ (br s, $1 \mathrm{H}$, $\mathrm{HO}, \mathrm{D}_{2} \mathrm{O}$ exchangeable), $8.45(\mathrm{~s}, 1 \mathrm{H}, 8-\mathrm{H}), 9.16$ (br s, $1 \mathrm{H}, \mathrm{HO}, \mathrm{D}_{2} \mathrm{O}$ exchangeable), $13.45\left(\mathrm{~s}, 1 \mathrm{H}, \mathrm{HO}, \mathrm{D}_{2} \mathrm{O}\right.$ exchangeable). ${ }^{13} \mathrm{C}$ NMR (75 MHz, DMSO-d6): $\delta=$ $20.64\left(\mathrm{CH}_{3}\right), 45.52,66.13,107.05,111.53,117.57$, $119.45,123.82,130.17,131.99,132.69,137.77,139.18$ (8-C), 148.54, 148.93, 149.36, 152.66, 158.86 (2-C). Anal. calcd for $\mathrm{C}_{22} \mathrm{H}_{21} \mathrm{~N}_{5} \mathrm{O}_{4} \cdot 0.5 \mathrm{H}_{2} \mathrm{O}: \mathrm{C}, 61.68 ; \mathrm{H}, 5.14$; $\mathrm{N}, 16.36$. Found: C, 61.75; H, 5.06; N, 16.20. 